\title{
Alveolar levels of immuno-inflammatory mediators in diffuse alveolar hemorrhage after allogeneic transplant
}

\author{
Lisa K. Vande Vusse ${ }^{1,2} \cdot$ Mark M. Wurfel $^{1} \cdot$ David M. Madtes ${ }^{1,2} \cdot$ H. Gary Schoch ${ }^{2}$ Susanna Harju-Baker $\mathbb{D}^{1}$. \\ Joshua A. Hill $\mathbb{D}^{2,3} \cdot$ Keith R. Jerome $e^{2,3} \cdot$ Michael Boeckh ${ }^{2,3} \cdot$ Timothy R. Watkins ${ }^{1}$
}

Received: 2 November 2017 / Revised: 25 November 2017 / Accepted: 9 February 2018 / Published online: 18 April 2018

(c) Macmillan Publishers Limited, part of Springer Nature 2018

Idiopathic pneumonia syndrome (IPS) is a severe and morbid complication of hematopoietic cell transplantation (HCT) that largely lacks effective treatments. The syndrome is defined as abnormal pulmonary physiology with multilobar opacities on chest imaging that are not attributable to lower respiratory tract infection (LRTI) or other organ failures [1]. Many pathologies meet IPS criteria, including organizing pneumonia, interstitial pneumonitis, and diffuse alveolar damage, among others. Biologic heterogeneity within this broadly defined syndrome may diminish the ability to detect the effect of treatments. An improved understanding of IPS pathobiology may catalyze the development of effective preventive and therapeutic strategies [2].

Several pro-inflammatory cytokines/chemokines are elevated in IPS [3, 4]. These biomarkers may be useful to distinguish IPS subphenotypes with unique biologic features. As a preliminary examination of this concept, we tested the hypothesis that immuno-inflammatory mediators measured in the blood and alveolar compartments of people with IPS differ according to the presence or absence of diffuse alveolar hemorrhage (DAH). DAH is a variably present manifestation of IPS that is proposed to have distinct biology [5]. We retrospectively studied 17 adults who underwent clinical bronchoscopy for diffusely abnormal chest imaging within 120 days of allogeneic HCT at Fred Hutchinson Cancer Research Center (FHCRC) between

Lisa K. Vande Vusse

lkvandev@u.washington.edu

1 Division of Pulmonary and Critical Care, University of Washington, Seattle, WA, USA

2 Clinical Research Division, Fred Hutchinson Cancer Center Research Center, Seattle, WA, USA

3 Vaccine and Infectious Disease Division, Fred Hutchinson Cancer Research Center, Seattle, WA, USA
2008 and 2010. None received specific tumor necrosis factor (TNF)- $\alpha$ inhibitors prior to bronchoscopy. Data and samples were banked prospectively with subjects' consent under FHCRC protocol 999.209 and were approved for use in this research (FHCRC protocols 808 and 1829/substudy 050).

We reviewed pertinent progress notes, microbiology reports, pathology reports, bronchoscopy reports, and radiographic images to identify IPS cases according to the American Thoracic Society definition [1]. We defined abnormal respiratory physiology as oxygen saturation $\leq 92 \%$ by pulse oximetry or arterial blood gas while breathing ambient air or supplemental oxygen. We defined LRTI as bronchoalveolar lavage fluid (BALF) growing any amount of Gram-negative rods, Mycoplasma spp., Chlamydophila spp., fungi/mold, Legionella, Nocardia, Mycobacteria, or $>10^{4}$ colony-forming units per $\mathrm{mL}$ of a single pathogenic Gram-positive coccus. Cytomegalovirus (CMV) infection required both culture and PCR positivity. We also classified as LRTI: any positive direct fluorescence antibody stain or $>40$ PCR copies of respiratory viruses (such as adenovirus, coronavirus, parainfluenza, influenza $\mathrm{A} / \mathrm{B}$, respiratory syncytial virus, human metapneumovirus, herpes simplex, and varicella zoster); positive stain for Pneumocystis jirovecii; and BALF or serum galactomannan $>0.5 \mathrm{ng} / \mathrm{L}$. DAH required documentation of increasingly bloody return from serial bronchoalveolar lavages, or report of "bloody" BALF followed by treatment with systemic corticosteroids for clinically diagnosed DAH.

According to local protocols, blood samples were drawn at weekly intervals to survey for CMV and processed and cryopreserved immediately using local standard operating procedures. When available, we selected blood samples collected prior to bronchoscopy or within 2 days of bronchoscopy to best reflect biologic activity at the time of bronchoscopy. BALF samples were obtained using standard clinical techniques and cryopresevered without processing. We thawed samples on an ice batch and centrifuged them at 
$1000 \times g$ for $10 \mathrm{~min}$ to remove debris. We then batchanalyzed in duplicate a broad array of immunoinflammatory cytokines, chemokines, and growth factors using commercially available multiplexed immunoassay kits per manufacturer guidelines (Meso Scale Diagnostics $^{\mathrm{TM}}$, Rockville, MD). We generated standard curves using serial dilutions of standard sample matrices of known concentration and determined analyte concentrations using Meso Scale Discovery Workbench version 4.0 (copyright 2007).

Individual analytes with a concentration $0.5 \mathrm{SD}$ or less above the mean signal for the blank control were replaced with a value of $0 \mathrm{pg} / \mathrm{mL}$ (analyte $n=149$ ). Analytes with a signal between 0.5 and $2.5 \mathrm{SD}$ above the mean signal for the blank were left unchanged. We performed two-way analysis of variance, and based on intra-class correlation coefficients $<0.7$, interleukin (IL)-2, IL-12 p70, IL-4, and TNF- $\beta$ were dropped for both BALF and plasma. In addition, IL-5, IL-10, IL-13, Macrophage-Derived Chemokine (MDC), and Thymus and activation-regulated chemokine (TARC) were dropped for BALF and TNF- $\alpha$ was dropped for plasma. We summarized concentrations of replicate samples as means and used two-sample Wilcoxon rank-sum tests for statistical comparisons. The statistical analysis was planned and performed by research team members with relevant expertise (Vande Vusse, Wurfel; computer code requests can be addressed to the corresponding author). We used StataIC 12.0 software (StataCorp LP, College Station, TX) for data analysis and defined statistical significance as a two-sided, $P<0.05$.

Eleven subjects met criteria for IPS - six with DAH and five without. Five subjects had evidence of LRTI. We excluded one subject with congestive heart failure from all analyses. Several conditioning regimens were used at the clinical providers' discretion. Mortality was high in all groups (Table 1).

Cytokine and chemokine levels were highly variable (Table 2). BALF concentrations of IL-15, MIP-1 $\alpha$ (CCL3), MIP-1 $\beta$ (CCL4), and MCP-1 were higher in subjects with IPS and DAH than those with non-DAH IPS. No blood biomarker differed between these groups (data not shown). The biomarkers elevated in BALF from subjects with DAH mediate the recruitment and activation of innate and adaptive immune effector cells [6-10]. Our findings are consistent with higher levels of immune system activity in the alveolar compartment of people with IPS and DAH than those with non-DAH IPS. DAH may be the clinical manifestation of unique lung-localized pathobiology that would not be recognized measuring blood biomarkers alone.

Given the number of comparisons made in our broad examination of biomarkers, some of our statistically significant findings may be false positives. Both preclinical and clinical studies have contributed importantly to the current
Table 1 Subject, stem cell transplant, and sampling characteristics

\begin{tabular}{|c|c|c|c|}
\hline Characteristic $^{\mathrm{a}}$ & $\begin{array}{l}\text { LRTI } \\
(n=5)\end{array}$ & $\begin{array}{l}\text { IPS with } \\
\text { DAH }(n=6)\end{array}$ & $\begin{array}{l}\text { IPS without } \\
\text { DAH }(n=5)\end{array}$ \\
\hline Age (years), mean (SD) & $45(15)$ & $37(7)$ & $49(9)$ \\
\hline Male & $4(80)$ & $4(67)$ & $3(60)$ \\
\hline White race & $4(80)$ & $2(33)$ & $4(80)$ \\
\hline Acute leukemia & $3(60)$ & $3(50)$ & $4(80)$ \\
\hline $\begin{array}{l}\text { Myeloablative } \\
\text { conditioning }\end{array}$ & $3(60)$ & $4(67)$ & $4(80)$ \\
\hline $\begin{array}{l}\text { Any total } \\
\text { body irradiation }\end{array}$ & $3(60)$ & $2(33)$ & $3(60)$ \\
\hline \multicolumn{4}{|l|}{ Stem cell source } \\
\hline Bone marrow & $2(40)$ & $3(50)$ & $1(20)$ \\
\hline Peripheral blood & $1(20)$ & $2(33)$ & $3(60)$ \\
\hline Cord blood & $2(40)$ & $1(17)$ & $1(20)$ \\
\hline \multicolumn{4}{|l|}{$\begin{array}{l}\text { HLA match and donor } \\
\text { relation }\end{array}$} \\
\hline Matched, related & 0 & $2(33)$ & $1(20)$ \\
\hline Matched, unrelated & $4(80)$ & $2(33)$ & $2(40)$ \\
\hline Mismatched & $1(20)$ & $2(33)$ & $2(40)$ \\
\hline Engrafted before bronch & $2(40)$ & $2(33)$ & $3(60)$ \\
\hline GVHD before bronch & $3(60)$ & $2(33)$ & $2(40)$ \\
\hline $\begin{array}{l}\text { Days HCT to bronch, } \\
\text { mdn (IQR) }\end{array}$ & $\begin{array}{l}20 \\
(19-36)\end{array}$ & $14(9-19)$ & $34(24-59)$ \\
\hline $\begin{array}{l}\text { Days bronch to blood } \\
\text { sample, mdn (IQR) }\end{array}$ & $1(1-1)$ & $5(2-7)$ & $1(1-1)$ \\
\hline $\begin{array}{l}\text { Died } \leq 90 \text { days from } \\
\text { bronch }\end{array}$ & $4(80)$ & $3(50)$ & $2(40)$ \\
\hline
\end{tabular}

LRTI lower respiratory tract infection, IPS idiopathic pneumonia syndrome, $D A H$ diffuse alveolar hemorrhage, $H L A$ human leukocyte antigen, GVHD acute graft-versus-host disease, bronch bronchoscopy

${ }^{a}$ Values are number of cases with percents in parentheses, unless otherwise noted

understanding of IPS. Reassuringly, our results are similar to those found in experimental models and clinical settings. Panoskaltsis-Mortari et al. examined lung protein extracts, serum, and BALF from a murine IPS model exposed to cyclophosphamide, total body irradiation, allogeneic HCT, and natural killer (NK)-cell depleted spleen cell infusion (a T-cell source) [11]. This study demonstrated time-varying elevations in MIP- $1 \alpha$ and MCP-1 messenger RNA expression and protein production in all compartments relative to healthy controls. In another preclinical study, MIP-1 $\alpha$, MIP$1 \beta$, and MCP-1 were expressed early after irradiated mice received allogeneic bone marrow transplants [12]. Experimental disruption of either MCP-1 or its receptor lowered BALF monocyte, macrophage, and in the latter case, T-cell counts and lessened histopathologic lung injury. Clinical studies demonstrate that MCP-1 levels are elevated in BALF collected from people with IPS relative to healthy controls and individuals with chronic noninfectious lung 
Table 2 Concentrations of immuno-inflammatory markers in bronchoalveolar lavage fluid

\begin{tabular}{|c|c|c|c|c|c|}
\hline & \multicolumn{2}{|c|}{$\begin{array}{l}\text { IPS with DAH } \\
(n=6)\end{array}$} & \multicolumn{2}{|c|}{$\begin{array}{l}\text { IPS without DAH } \\
(n=5)\end{array}$} & \multirow[b]{2}{*}{$P$} \\
\hline & $\begin{array}{l}\text { Median } \\
(\mathrm{pg} / \mathrm{mL})\end{array}$ & IQR & $\begin{array}{l}\text { Median } \\
(\mathrm{pg} / \mathrm{mL})\end{array}$ & IQR & \\
\hline IFN- $\gamma$ & 12.8 & 24.3 & 0.5 & 4.3 & 0.07 \\
\hline IL-12 p 40 & 5.6 & 28.1 & 2.5 & 2.0 & 0.36 \\
\hline IL-17A & 3.8 & 10.9 & 0.0 & 0.0 & 0.07 \\
\hline IL-13 & 10.5 & 8.4 & 7.8 & 2.7 & 0.58 \\
\hline TNF- $\alpha$ & 4.1 & 31.4 & 1.6 & 0.5 & 0.27 \\
\hline IL-1 $\beta$ & 3.2 & 5.2 & 1.8 & 1.4 & 0.27 \\
\hline IL-6 & 2933.0 & 3335.5 & 77.7 & 2169.8 & 0.07 \\
\hline IL-8 & 1785.1 & $19,426.0$ & 249.2 & 167.3 & 0.10 \\
\hline MIP- $1 \alpha$ & 96.7 & 222.2 & 21.1 & 17.7 & 0.02 \\
\hline MCP-1 & 6402.3 & 4850.1 & 612.7 & 2251.2 & 0.01 \\
\hline GM-CSF & 49.3 & 137.7 & 5.0 & 25.3 & 0.10 \\
\hline Eotaxin & 88.0 & 185.8 & 52.8 & 69.7 & 0.36 \\
\hline Eotaxin-3 & 9.7 & 11.2 & 1.8 & 5.3 & 0.10 \\
\hline IL-15 & 14.6 & 18.7 & 5.9 & 5.3 & 0.03 \\
\hline IL-16 & 188.6 & 183.3 & 181.9 & 104.6 & 0.86 \\
\hline IL-1 $1 \alpha$ & 5.9 & 8.2 & 2.2 & 2.6 & 0.36 \\
\hline IL-7 & 1.5 & 5.3 & 1.9 & 6.4 & 0.58 \\
\hline IP-10 & 305.5 & 529.1 & 57.4 & 1645.0 & 0.92 \\
\hline MIP-1ß & 213.9 & 373.0 & 15.4 & 6.0 & 0.03 \\
\hline VEGF & 89.3 & 34.5 & 77.2 & 28.9 & 1.00 \\
\hline MCP-4 & 170.2 & 1607.1 & 25.0 & 232.0 & 0.25 \\
\hline
\end{tabular}

disease after HCT [4, 12]. In one observational study, BALF sampled before and after TNF- $\alpha$ antagonist therapy demonstrated decreases in MCP-1 and other inflammatory mediators in the subset of subjects who experienced clinical improvement [4]. Our study extends these findings by suggesting that IPS subtypes may have different inflammatory profiles and therefore differing response to immunomodulatory therapies.

A recent study applied modern molecular diagnostic techniques to BALF samples from individuals previously diagnosed with IPS and found occult viruses of unknown clinical significance [13]. In a post hoc analysis, we examined the distribution of the most prevalent occult virus known to be a human pathogen, HHV-6, using a real-time quantitative fluorescent probe PCR assay as previously described [14, 15]. Eight of the 17 study subjects were HHV-6 positive. HHV-6 was similarly distributed between subjects with and without IPS and between subjects with IPS and DAH and subjects with non-DAH IPS. BALF and plasma cytokine levels did not differ between IPS cases with HHV-6 and IPS cases without HHV-6 (data not shown). Although it is possible that the current IPS definition misclassifies some infections, HHV-6 did not clearly drive the findings of our study. Future research must consider the potential role of "occult" viral infection when defining IPS subphenotypes.

This research has several potential limitations. This small study lacking at-risk controls may be underpowered to detect differences. The lack of standard methods to correct for dilution of BALF may introduce error. The impacts of therapeutic corticosteroids, the cryopreservation of unprocessed BALF, and variable timing of sample collection on our results are unknown. The onset of non-DAH IPS occurred later than IPS with DAH and more often after engraftment. In this study, DAH may be a surrogate for important biologic differences between early- and lateronset IPS. Alternatively, the differences we found may relate to time passing instead of varied biology. A confirmatory prospective validation study is needed that ideally collects and processes plasma and BALF simultaneously. Analysis of protein levels, leukocyte expression, and cellular profiles may help elucidate important biologic differences between IPS subtypes.

In conclusion, the immune system is particularly active in the DAH phenotype of IPS occurring within 120 days of allogeneic HCT. The relevance of this small study lies in its potential to influence future research. Our findings support the hypothesis that IPS includes a spectrum of pathologies that may differentially respond to risk factor modification and therapies. The heterogeneous nature of the current IPS definition may not allow research to reveal the true effects of immune modulating therapies. Future research is needed to identify biologically distinct IPS subphenotypes as progress toward precision medicine and improved outcomes.

Acknowledgements We acknowledge the contributions of Terry Stevens-Ayers and Jessica Yi for their assistance obtaining all study samples from the Infectious Disease Sciences Biospecimen Repository, Vaccine and Infectious Disease Division, Fred Hutchinson Cancer Research Center.

Funding This research was funded by NHLBI Institutional Training Grant 2 K12 HL087165-06 (PI Janis Abkowitz, MD), K24HL093294 (PI M.B.), and research funds from BloodWorks Northwest, Seattle, WA. At the time of this research, T.R.W. was supported by a grant from the NIGMS K23GM08672.

\section{Compliance with ethical standards}

Conflict of interest T.R.W. currently works for Gilead Sciences, Inc. The remaining authors declare that they have no conflict of interest.

\section{References}

1. Panoskaltsis-Mortari A, Griese M, Madtes DK, Belperio JA, Haddad IY, Folz RJ, et al. An official American Thoracic Society research statement: noninfectious lung injury after hematopoietic 
stem cell transplantation: idiopathic pneumonia syndrome. Am J Respir Crit Care Med. 2011;183:1262-79.

2. Radhakrishnan SV, Hildebrandt GC. A call to arms: a critical need for interventions to limit pulmonary toxicity in the stem cell transplantation patient population. Stem Cell Transplant. 2015;10:8-17.

3. Clark JG, Madtes DK, Martin TR, Hackman RC, Farrand AL, Crawford SW. Idiopathic pneumonia after bone marrow transplantation: cytokine activation and lipopolysaccharide amplification in the bronchoalveolar compartment. Crit Care Med. 1999;27:1800-6.

4. Yanik GA, Ho VT, Levin JE, White ES, Braun T, Antin JH, et al. The impact of soluble tumor necrosis factor receptor etanercept on the treatment of idiopathic pneumonia syndrome after allogeneic hematopoietic stem cell transplantation. Blood. 2008;112:3073-81.

5. Afessa B, Tefferi A, Litzow MR, Krowka MJ, Wylam ME, Peters SG. Diffuse alveolar hemorrhage in hematopoietic stem cell transplant recipients. Am J Respir Crit Care Med. 2002;166:641-5.

6. Uguccioni M, D'Apuzzo M, Loetscher M, Dewald B, Baggiolini $\mathrm{M}$. Actions of the chemotactic cytokines MCP-1, MCP-2, MCP-3, RANTES, MIP-1 alpha and MIP-1 beta on human monocytes. Eur J Immunol. 1995;25:64-8.

7. Schall TJ, Bacon K, Camp RDR, Kaspari JW, Goeddel DV. Human macrophage inflammatory protein $\alpha$ (MIP-1 $\alpha)$ and MIP-1 $\beta$ chemokines attract distinct populations of lymphocytes. J Exp Med. 1993;177:1821-5.

8. Maghazachi AA, Al-Aoukaty A, Schall TJ. C-Cchemokines induce the chemotaxis of NK and IL-2-activated NK cells. Role for G proteins. J Immunol. 1994;153:4969-77.
9. Mrozek E, Anderson P, Caligiuri MA. Role of interleukin-15 in the development of human CD56+ natural killer cells from CD34+ hematopoietic progenitor cells. Blood. 1996;87: 2632-40.

10. Cooke KR, Coghill JM, Hildebrandt GC, Serody JS. Chemokines and graft-versus-host disease. In: Socié G, Blazar BR, editors. Immune biology of allogeneic hematopoietic stem cell transplantation: models in discovery and translation. 1st ed. Amsterdam: Academic Press; 2013. p. 393-424.

11. Panoskaltsis-Mortari A, Strieter RM, Hermanson JR, Fegeding KV, Murphy WJ, Farrell CL, et al. Induction of monocyte- and Tcell-attracting chemokines in the lung during generation of idiopathic pneumonia syndrome following murine bone marrow transplantation. Blood. 2000;96:834-9.

12. Hildebrandt GC, Duffner UA, Okiewicz KM, Corrion LA, Willmarth NE, Williams DL, et al. A critical role for CCR2/MCP-1 interactions in the development of idiopathic pneumonia syndrome after allogeneic bone marrow transplantation. Blood. 2004;103:2417-26.

13. Seo S, Renaud C, Kuypers JM, Chiu CY, Huang ML, Samayoa E, et al. Idiopathic pneumonia syndrome after hematopoietic cell transplantation: evidence of occult infectious etiologies. Blood. 2015;125:3789-97.

14. Zerr DM, Gupta D, Huang M-L, Carter R, Corey L. Effect of antivirals on human herpesvirus-6 replication in hematopoietic stem cell transplant recipients. Clin Infect Dis. 2002;34: 309-17.

15. Hill JA, HallSedlak R, Magaret A, Huang M-L, Zerr DM, Jerome $\mathrm{KR}$, et al. Efficient identification of inherited chromosomally integrated human herpesvirus- 6 using specimen pooling. J Clin Virol. 2016;77:71-6. 